\title{
Helicobacter Pylori Infection And Gastric Cancer: Is It Our National Problem?
}

\author{
MM RAHMAN $^{\mathrm{a}}, \mathrm{S}^{\mathrm{S}} \mathrm{ALAM}^{\mathrm{b}}$, AMK IQBAL $^{\mathrm{c}}$, MM HOSSAIN $^{\mathrm{d}}$, AMK SARKER $^{\mathrm{e}}$, M ISLAM $^{\mathrm{f}}$
}

\begin{abstract}
Summary:
Gastric cancer is a leading cause of cancer death worldwide. In Bangladesh it ranks a leading position among the cancers patients. A large body of evidence supports a causal role of Helicobacter pylori in the majority of gastric malignancies. Scientists throughout the world explored and reached to the understanding about the pathogenesis of their relationship, but much remains to be learned. Moreover, because of the high prevalence of infection, the lack of definitive trials, and the challenges of $\mathrm{H}$. pylori treatment, there remains a debate
\end{abstract}

\section{Introduction:}

Despite many preventive measures and screening steps in many parts of the world, gastric Cancer(GC) is still now a leading cause of cancer-related mortality, causing $9.7 \%$ of all cancer-related deaths around the world. ${ }^{1}$ Almost one million new cases of stomach cancer were estimated to have occurred in 2012 (952,000 cases, $6.8 \%$ of the total), making it the fifth most common malignancy in the world, after cancers of the lung, breast, colorectum and prostate. ${ }^{2}$ More than $70 \%$ of cases $(677,000$ cases) occur in developing countries (456,000 in men, 221,000 in women), and half the worlds' total occurs in Eastern Asia (mainly in

a. Prof. M Mizanur Rahman, Former Professor Surgical Oncology, National Institute of Cancer Research and Hospital, Mohakhali, Dhaka.

b. Dr. Shahjadul Alam, Assistant Professor Surgical Oncology, National Institute of Cancer Research and Hospital, Mohakhali, Dhaka

c. Dr. Abu Mohammad Khaled Iqbal, Assistant Registrar Casualty Surgery, Comilla Medical College, Comilla.

d. Dr. Md. Monoar Hossain, Assistant Professor Surgical Oncology, Khulna Medical College, Khulna.

e. Dr.Abu Mohammad Kawser Sarker, Assistant Professor Surgery, Aichi Medical College, Uttara, Dhaka

f. Dr. Monjurul Islam, Assistant Registrar Surgical Oncology, National Institute of Cancer Research and Hospital, Mohakhali, Dhaka

Address of Correspondence: Prof M Mizanur Rahman, House-7/6, Block B, Lalmatia, Dhaka.Email: mizannicrh@gmail.com

Received: 13 June 2017

Accepted: 1 March 2018 regarding the consensus on the role of routine screening and treatment of this infection to prevent cancer. This article reviews the current knowledge on $\mathrm{H}$. pylori and its role for gastric cancer, present status of Bangladesh and a recommendation for reduction of the infectivity among the common population.

Key words: H pylori, Infection, Gastric cancer.

(J Bangladesh Coll Phys Surg 2018; 36: 70-76) DOI: http://dx.doi.org/10.3329/jbcps.v36i2.36069

China. ${ }^{2}$ Though Bangladesh is lacking a population based statistics or national cancer registry for cancers, there are very few hospital based statistics. According to the reports from the national guideline on gastric cancer management it is ranking as the fifth most common cancer and third most common among the males. From the unpublished data across the country from different medical institutions it has been estimated that GC possesses second position after lung cancer in males. ${ }^{3}$

Helicobacter pylori (H pylori) infection is also considered to be the main risk factor of gastric cancer development among all the environmental factors, namely gastric carcinoma and gastric mucosaassociated lymphoid tissue lymphoma. ${ }^{4}$ This paper reviews the characteristics of $\mathrm{H}$. pylori and the consequences of the infection linking with gastric cancer, its status in Bangladeshi patients and rationality of making a national programme for eradication of $\mathrm{H}$. pylori in the community.

\section{Stomach cancer and its epidemiology}

Stomach cancer prevails about twice as high in men as in women and vary widely across countries. In general, incidence rates are highest in Eastern Asia (particularly in Korea, Mongolia, Japan, and China), Central and Eastern Europe, and South America and lowest in Northern America and most parts of Africa. Regional variations in part reflect differences in dietary patterns, food storage, and the availability of fresh produce, as well as the prevalence of Helicobacter pylori infection. Chronic infection with $\mathrm{H}$. pylori is the strongest 
identified risk factor for stomach cancer, with about $90 \%$ of new cases of noncardia gastric cancer worldwide attributed to this bacteria. ${ }^{5}$

A steady decline in stomach cancer incidence and mortality rates has been observed in the developed countries in Northern America and Europe since the middle of the 20th century. Similar decreasing trends have been noted in more recent years in areas with historically high rates, including several countries in Asia (Japan, China, and Korea), Latin America (Colombia and Ecuador), and Europe (Ukraine).Factors that have contributed to these declines are thought to include the increased availability of fresh fruits and vegetables, decreased reliance on salt preserved foods, and reduction in chronic $\mathrm{H}$. pylori infection due to improved sanitation and antibiotics. ${ }^{6}$

\section{Helicobacter pylori}

H. pylori is a Gram-negative, spiral-shaped bacterium that is characterized by its many unipolar flagella, which give it corkscrew-like motility, and its unique production of urease. Among bacteria, it finds a niche in both the antral and fundic mucosa of the stomach under the mucus gel. The presence of infection is universally associated with chronic and acute inflammation and, more variably, with other gastric lesions, including lymphoid follicles, atrophic gastritis and intestinal metaplasia. Treatment with antimicrobial agents causes inflammation to regress over time. ${ }^{7}$ With relapse of infection, the gastritis is again observed ${ }^{8}$

H. pylori is typically acquired during childhood and causes lifelong infection thereafter. ${ }^{9}$ Although previously almost universal in humans, currently 'only' half of the world's population is infected with $\mathrm{H}$. pylori. Transmission is largely from person to person via the faecal-oral or the gastric-oral route within families, particularly in settings of poor sanitation and hygiene ${ }^{10}$ The prevalence of infection varies worldwide, with continued hyper-endemicity in developing countries but a markedly lower prevalence in developed countries. ${ }^{11} \mathrm{H}$. pylori is now rare in native-born and middle- or upper-class children of Western Europe, ${ }^{12}$ North America, ${ }^{13}$ Oceania ${ }^{14}$ and Japan. ${ }^{15,16}$

\section{Epidemiological Links between Gastric Cancer and $\mathrm{H}$. pylori:}

It has been 30 years since the discovery of Helicobacter pylori (H. pylori) in 1983 by Australian physicians Robert Warren and Berry Marshal. ${ }^{17}$ In view of the various epidemiological studies worldwide, the International Agency of Cancer classified H. pylori as a Class '! carcinogen for gastric cancer in $1994 .{ }^{18}$ Since then the bacterium is thought to be one of the causative factors in the development of gastric cancer. H. pylori is a gastric pathogen that colonizes approximately $50 \%-60 \%$ of the world's population. Infection with $H$. pylori causes chronic inflammation and significantly increases the risk of developing duodenal and gastric ulcer disease and gastric cancer. Studies in Asian countries such as Thailand, India, Bangladesh, Pakistan, Iran, Saudi Arabian countries, Israel and Malaysia, have reported a high frequency of H. pylori infection co-existing with a low incidence of gastric cancer. ${ }^{19,20}$

Subsequent to its discovery in the early 1980 s, over 1000 studies have been conducted on $\mathrm{H}$. pylori and its association with cancer, including observational studies (ecological, case-control and cohort), clinical trials of H. pylori eradication, pathological studies and animal models. The results have been overwhelmingly in favour of a link between infection and malignancy. Among the many observational studies in humans, case-control studies indicate the lowest risk for cancer (1.8-fold increase). ${ }^{21}$ It is now understood that these studies underestimate the true risk due to loss of $\mathrm{H}$. pylori as the mucosa undergoes malignant transformation.

A number of case-control studies indicate higher relative risks in meta-analyses. ${ }^{22}$ However, the most compelling observational evidence of an association between $\mathrm{H}$. pylori infection and gastric cancer comes from longitudinal cohort studies. In a large prospective trial conducted in Japan, 36 out of 1246 infected individuals developed gastric cancer compared to none of 280 uninfected participants. They finally concluded that persons with $H$. pylori infection and nonulcer dyspepsia, gastric ulcers, or gastric hyperplastic polyps are also at risk, but those with duodenal ulcers are not. ${ }^{23}$ A prospective study of 1225 Taiwanese patients confirmed this finding that all gastric malignancies, including adenocarcinoma and lymphoma, developed in H. pylori-infected patients. The finding implies that H. pylori is a necessary cause of most gastric malignancies. Multivariate analysis showed in their study that intestinal metaplasia was the only independent factor predicting subsequent development 
of gastric malignancy in $\mathrm{H}$. pylori-infected subjects with an odds ratio of 4.5 (95\% CI 1.1-19.1). ${ }^{24}$

Not all H. pylori are alike, however, and the epidemiological story is complex. Individuals with antibodies to H. pylori's CagA protein (a marker for the more inflammatory and virulent strain bearing a pathogenicity island of genes) have a particularly high risk of cancer. A meta-analysis of studies shows that CagA-positive strains increase the risk of noncardia gastric cancer two-fold compared to CagA-negative strains. ${ }^{25}$ Moreover, gastric cancer, similar to H. pylori, is heterogeneous, with two histological types predominating: the intestinal-type and the diffuse type. Although H. pylori has been linked to both histological types, CagA appears to enhance the risk of the intestinal type that arises in the setting of inflammation, atrophic gastritis and intestinal metaplasia, but not the risk of the diffuse type that appears to stem from e-cadherin mutations. ${ }^{26}$

Based on the observational and experiment studies, the attributable risk of gastric cancer in the population has been estimated to be $75 \% .{ }^{27}$ If this is accurate, $\mathrm{H}$. pylori would be responsible for as many as $5.5 \%$ of all cancers, making it the leading infectious cause of cancer worldwide and second only to smoking as a defined cause of malignancy. ${ }^{16}$

\section{$H$ pylori and Mechanisms of Gastric Carcinogenesis:}

Gastric adenocarcinoma is a heterogeneous cancer. First, it is necessary to distinguish the tumours arising from the gastric proximal stomach (cardia), as most of them are not linked to H.pylori infection from those found in the distal part of the stomach. Among tumours from the distal stomach, on the basis of histology, it is usual to differentiate two types of cancer lesions: the intestinal type and the diffuse type according to the Lauren classification. ${ }^{28}$ Intestinal type cancer is the most frequent. It corresponds to a slow evolution of the gastric mucosa which becomes atrophic; then intestinal metaplasia appears, followed by dysplasia and ultimately in situ gastric carcinoma and metastatic carcinoma. This is the so-called Correa cascade, which was described before H. pylori was discovered and appears late in life.$^{29}$ The other histologic type of gastric carcinoma is the diffuse type, which does not show these different steps and usually occurs early in life. Furthermore, mutations in the E-cadherin gene (CDH1) are found in about $30 \%$ of the cases. The expression of this molecule is then inhibited at the adherent cellular junctions, leading to invasive tumours. Besides this histologic classification, a molecular classification has recently been proposed. ${ }^{28}$

Literature is showing that mechanisms of $\mathrm{H}$. pyloriinduced carcinogenesis are in the phase of understanding, inflammation is the most commonly cited factor in the carcinogenic process. Inflammation is thought to induce cancer by increasing production of free radicals ${ }^{30}$, increasing apoptotic and necrotic epithelial cell death and augmenting cell proliferation .${ }^{31}$ To compound these pro-carcinogenic processes, $\mathrm{H}$. pylori has been noted to reduce DNA repair in vivo and in vitro. The importance of inflammation as a risk factor is supplemented by three complementary observations: first, that the bacterial strains that induce the most inflammation are most closely linked to malignancy ${ }^{32}$; second, that pro-inflammatory host cytokine polymorphisms increase cancer risk, and third, that nonsteroidal anti-inflammatory agents appear to decrease the risk of cancer. ${ }^{33}$

Most recently, much attention has been given to the relationship between $\mathrm{H}$. pylori, stem cells and cancer. Some have proposed that $\mathrm{H}$. pylori preferentially damages parietal cells, thereby altering the maturation process of epithelial stem cells. ${ }^{34}$ Others report that inflammation related to $\mathrm{H}$. pylori recruits peripheralor bone-marrow derived stem cells to the gastric mucosa, which then transform into the malignant clone. The strongest evidence of this comes from experiments performed by Houghton et al..$^{35}$ who identified bonemarrow derived stem cells as the cells of malignant origin in C57BL/6 mice. By contrast, Giannakis et al ${ }^{36}$ reported identifying $\mathrm{H}$. pylori inside gastric stem cells. They further observed that an isolate from a cancer patient had closer affinity to gastric stem cells, causing more profound regulation of cell function, than did an isolate from the same patient 4 years before the cancer diagnosis. ${ }^{16}$

It is well proven that cancer is fundamentally a result of genetic instability. A small proportion of gastric cancers are familial and related to inherited genetic abnormalities that involve alterations in tumour suppressor genes, proto-oncogenes, gatekeeper genes, enzymes, growth factors and membrane or nuclear 
receptor. ${ }^{37}$ Studies are evidencing that chronic $H$. pylori infection causes lifelong acute and chronic gastric inflammation which can result in DNA damage and genetic instability. ${ }^{38}$ Recently, it has been recognized that the $H$. pylori organism can also cause genetic instability, including double-stranded DNA breaks and can produce gene activation and silencing via epigenetic pathways. ${ }^{38}$ The pathogenesis of $H$. pylori -related genetic instability is complex and as yet incompletely understood with both inflammationinduced reactive oxygen species and reactive nitrogen species playing important roles. ${ }^{39}$ Although $H$. pylori induces lifelong gastric mucosal inflammation, gastric cancer is not a preordained outcome. The clinical manifestations of the infection vary regionally, with important host, H. pylori strain, and environmental factors all interacting to determine the outcome for a particular patient and region. Since $H$. pylori is a necessary cause of gastric cancer, a high incidence of gastric cancer requires a high prevalence of $H$. pylori . However, even among high $H$. pylori prevalence societies such as China, there are strong geographic differences in the incidence of gastric cancer. ${ }^{40}$

\section{H. pylori infection status in Bangladesh:}

Bangladesh is a South Asian developing country, where the rate of $H$. pylori infection is also high. In their serological study, Ahmad et al in 1997 reported that the prevalence of $H$. pylori in Bangladesh was $92 \% .{ }^{41}$ Mahalanabis et $\mathrm{al}^{42}$ in a study of $13 \mathrm{C}$-urea breath test also reported that the prevalence of $H$. pylori was $63 \%$ in infants aged 1-3 months, $33 \%$ in 10-15-month-old children, $84 \%$ in 6-9 years old. Moreover, the overall H. pylori prevalence in other Asian countries including, India ( $79 \%$ by ELISA), Pakistan ( $84 \%$ by PCR), and Japan (41\% by measuring urinary levels of anti-H. pylori antibody) was also reported high.12-14 In Europe (,40\%) and the United States (40\%), a significantly lower prevalence rate of $H$. pylori was observed. ${ }^{43,44}$ High $H$. pylori infection rates in developing countries compared to the developed world may be the consequence of poor socioeconomic conditions and unhygienic life styles. ${ }^{45}$

Until now, some studies have tried to show the prevalence of $H$. pylori infection in Bangladesh by serological methods, urea breath test, or CLO test. But there has not been less study to perform $H$. pylorispecific PCR directly on extracted DNA from gastric biopsies and CLO test together to determine H. pylori infection in our country. In one study in Bangladesh, for the first time PCR using H. pylori-specific $16 \mathrm{~S}$ rRNA primers along with CLO test in endoscopic biopsies to determine the incidence of $H$. pylori infection in was used. They found that among 111 patients, $60(54.05 \%)$ were positive by the CLO test and 54 (48.65\%) were positive by PCR. ${ }^{46}$

Gastric biopsies from 111 patients from gastroscopic biopsy at a hospital in Chittagong from July 2015 to November 2015 were collected. Total genomic DNA was extracted from the gastric biopsies by the phenol/ chloroform DNA extraction method.Molecular detection of $H$. pylori was then performed on extracted DNA from biopsies by PCR using primers to amplify a 109 product for the $H$. pylori 16S rRNA region. Among the biopsied samples, all the 74 cases being $H$. pylori positive for any of the two tests were considered for assessing the association between $H$. pylori infection and clinical presentations. It was observed that all the cases of duodenal ulcer had evidence of $H$. pylori infection, while patients with gastric ulcer had H. pylori in $75 \%$ of cases and the correlation between them was also proven to be statistically significant $(P=$ $0.05)$. Interestingly, dyspeptic patients with normal endoscopic findings had $H$. pylori in $87.5 \%$ of cases and had a significant association $(P=0.05)$ with $H$. pylori positive as well. ${ }^{46}$

In another study among the 181 subjects, 166 (92\%) had H. pylori specific antibodies and $15(8 \%)$ were seronegative. No significant difference $(p<0.90)$ in seroprevalence rates was observed among different age groups. $^{41}$

Recently in a case control study of 114 cases against 520 controls of the community it was shown that

significantly more patients in the case group (86.8\%) were found to be seropositive for $\mathrm{H}$. pylori antigen in contrast to the control group $(67.5 \%)$. All of the cases in the present study were in advanced stage of gastric cancer. Controls were endoscopically negative for any pathological lesion. It was noted that undifferentiated gastric carcinoma had slightly more association with H. pylori infection. Younger patients $(<40$ years of age) $H$. pylori infection had been found to be at higher relative risk for $\mathrm{GC}$ than older patients. ${ }^{46}$

Association of $\mathbf{H}$ pylori infection and genetic mutation in gastric cancer patients in Bangladesh: We could finally explore the status of p53 alteration which was remarkably present in our patients and had 
strong association with $\mathrm{H}$ pylori infection examined in an study done in national institute of cancer research and hospital, Mohakhali,,Dhaka. Unpublished data revealed after gene analysis that among the $\mathrm{H}$ pylori infected cases $80 \%$ have alteration of $\mathrm{p} 53$ in the tested gene in the current series of 71 gene analysis, despite of using only the 5 and 6 exons. Chi square and regression analysis shows that they have strong and significant association. Over $86 \%$ Patient of gastric cancer infected with $\mathrm{H}$ pylori had mutant $\mathrm{p} 53$ gene. Multigene analysis showed that over $88 \%$ of the $\mathrm{H}$ pylori infected patients had gene mutation.

In Bangladesh, gastric cancer incidence is in rising trend. Regarding $\mathrm{H}$ pylori infection, on the other hand different studies directs that in the last 20 years infection is in down trend in the globe. Overall study is showing that in Bangladesh $\mathrm{H}$. pylori is found in $>80 \%$ of GC cases. To date, existing findings indicate that $\mathrm{GC}$ is the biological translation of carrying an infectious disease, which is interestingly preventive with anti-H. pylori regimen. Therefore, as an inevitable consequence, identification of $\mathrm{H}$. pylori colonized in people with high risk of GC is the main direction of the future research. It is postulated that if $\mathrm{H}$. pylori can be removed from the population, it has been estimated that $<" 75 \%$ of GC would be eliminated. ${ }^{48}$

\section{Eradication:}

Effect of $H$. pylori eradication on cancer incidence The effect of $\mathrm{H}$. pylori eradication on reducing gastric cancer incidence is related to the risk existing at the time of eradication therapy. The major benefits for treatment of those at little or no cancer risk at the time of eradication include removal from the reservoir of infection responsible for spread within society, prevention of development of diseases caused by $\mathrm{H}$. pylori such as peptic ulcer disease and prevention of progression of gastritis with its associated risk of gastric cancer. Early studies of $\mathrm{H}$. pylori eradication in gastric cancer used mixed populations with varying degrees of cancer risk and were of relatively short duration. 49

A large-scale cohort study from Taiwan followed 80,000 patients with peptic ulcer for 10 years after $\mathrm{H}$. pylori eradication therapy. The patients were assigned to an early eradication group(patients underwent $\mathrm{H}$. pylori eradication therapy at the time of diagnosis) or a late eradication group (patients underwent H. pylori eradication therapy 1 year after diagnosis). The incidence of gastric cancer was markedly lower in the early eradication group than in the late eradication group suggesting that, while the effect of $\mathrm{H}$. pylori eradication therapy in reducing the incidence of gastric cancer is obvious, the earlier the eradication the better. Mass eradication of $\mathrm{H}$. pylori was started in Taiwan in 2004 and initially included 4121 subjects. Compared to the 5 year period before H.pylori therapy, the effectiveness of $\mathrm{H}$. pylori eradication therapy in reducing the incidence of gastric cancer was estimated to be $25 \%$ (rate ratio $0.753,95 \%$ confidence interval (CI) $0.372-1.525$ ) and the reduction in peptic ulcer disease $67.4 \%$ (95\% CI 52.2-77.8). ${ }^{50}$

So finally they demonstrated that mass eradication of $\mathrm{H}$ pylori infection was associated with a significant reduction in gastric atrophy within a relatively short study period, in parallel with an increase in gastro oesophageal reflux. Whether a meaningful reduction in gastric cancer can be achieved following the Correa pathway should be verified in a further long-term follow up study in this region, which has a high prevalence of $\mathrm{H}$ pylori infection and a high incidence of gastric cancer. ${ }^{51}$

In December 2013, a Working Group Meeting was hosted in Lyon, France by the International Agency for Research on Cancer (IARC) to review the accumulated evidence that supported the use of mass eradication of H. pylori as a strategy to prevent gastric cancer. On the basis of the favourable results from the randomized con-trolled trials (RCTs) and observational studies, the expert working group confirmed that this strategy was effective; a recommendation has been made to encourage health-care agencies to include such a strategy in national cancer control programs. In January 2014, a global consensus meeting was held in Kyoto, Japan to evaluate the management of $\mathrm{H}$. pylori-related gastritis, a precursor to gastric cancer. Similarly, consensus has been reached in the conclusion that eradication of $\mathrm{H}$. pylori can prevent gastric cancer and the recommendation that all carriers of $\mathrm{H}$. pylori should be treated to eradicate this pathogen. ${ }^{52}$

Taking consideration the limited data in Bangladesh it is to taken into account that in Bangladesh $\mathrm{H}$ pylori is also an important risk factor or a causative agent for gastric 
cancer. So it is the high time to think that national level or large scale eradication of $\mathrm{H}$ pylori is needed to combat the future incidences of gastric cancer.

\section{Conclusion:}

From the hundreds of studies including Bangladesh it has been seen that $\mathrm{H}$ Pylori is the leading causative agent for Gastric cancer, there are also documents to have genetic links in the process of carcinogenesis. In Bangladesh though very few studies are carried out in the last two decades including a case control study, all studies have evidence to have association of $\mathrm{H}$ pylori with gastric cancer. Internationality it is seen that there are some studies to carry out successful eradication therapy which resulted reduced incidences of the cancer. So it is the high time in Bangladesh to undertake the schemes at the national level for large scale eradication against the $\mathrm{H}$ pylori which might play a role for the reduced incidences of the deadly disease like gastric cancer.

\section{References:}

1. Ferro A, Peleteiro B, Malvezzi M, Bosetti C, Bertuccio P, Levi F,Negri E, La Vecchia C, Lunet N.Worldwide trends in gastric cancer mortality (1980-2011), with predictions to 2015, and incidence by subtype. Eur J Cancer 2014; 50: 1330-1344

2. www.http://globocan.iarc.fr/old/FactSheets/cancers/stomachnew.asp accessed on 12.08.2017

3. Gastric Cancer Management. National Guideline of of Bangladesh. 2014

4. International Agency for Research on Cancer. Schistosomes, liver flukes and Helicobacter pylori. IARC Monographs on the Evaluation of Carconogenic Risks to Humans 61. Lyon, France: IARC; 1994. 177-220. http://monographs.iarc.fr/ENG/ Monographs/vol61/index.php.

5. Plummer M, Franceschi S, Vignat J, Forman D, de Martel C. Global burden of gastric cancer attributable to pylori. Int J Cancer 2015; 136:487-490.

6. Parkin DM. The global health burden of infection-associated cancers in the year 2002. Int J Cancer 2006; 118:3030-3044

7. Ohkusa T, Fujiki K, Takashimizu I et al. Improvement in atrophic gastritis and intestinal metaplasia in patients in whom Helicobacter pylori was eradicated. Ann Intern Med 2001; 134: 380-386.

8. Patchett S, Beattie S, Leen E, Keane C, O’Morain C. Helicobacter pylori and duodenal ulcer recurrence. Am J Gastroenterol 1992; 87:24-27.

9. Rowland M, Daly L, Vaughan M, Higgins A, Bourke B, Drumm B. Age-specific incidence of Helicobacter pylori. Gastroenterology 2006; 130: 65-72; quiz 211.
10. Perry S, De La Luz Sanchez M, Shufang Y et al. Gastroenteritis and transmission of Helicobacter pylori infection in households. Emerg Infect Dis 2006;12: 1701-1708.

11. Brown LM. Helicobacter pylori: epidemiology and routes of transmission.Epidemiol Rev 2000; 22: 283-297.

12. Mourad-Baars PE, Verspaget HW, Mertens BJ, Mearin ML. Low prevalence of Helicobacter pylori infection in young children in the Netherlands. Eur J Gastroenterol Hepatol 2007; 19: 213-216.

13. Segal I, Otley A, Issenman R et al. Low prevalence of Helicobacter pylori infection in Canadian children: a cross-sectional analysis. Can J Gastroenterol 2008; 22: 485-489.

14. Hardikar W, Grimwood K. Prevalence of Helicobacter pylori infection in asymptomatic children. J Paediatr Child Health 1995; 31:537-541.

15. Okuda M, Miyashiro E, Booka M, Tsuji T, Nakazawa T. Helicobacter pylori colonization in the first 3 years of life in Japanese children. Helicobacter 2007;12: 324-327.

16. V. Herrera1 and J. Parsonnet..Helicobacter pylori and gastric adenocarcinoma.Clin Microb and Infect. 2009;15: 971-76.

17. Marshall BJ, Warren JR. Unidentified curved bacilli in the stomach of patients with gastritis and peptic ulceration. Lancet 1984;1:1311-1315.

18. Graham DY. Helicobacter pylori infection in the pathogenesis of duodenal ulcer and gastric cancer: a model. Gastroenterology 1997;113:1983-1991.

19. Malfertheiner P, Megraud F, O'Morain CA, et al., European Helicobacter Study Group. Management of elicobacter pylori infection-the Maastricht IV/Florence Consensus Report. Gut 2012;61:646-664.

20. Bor-Shyang S, Ming-Shiang W, Cheng-Tang C, Jing-Chuan L, Deng-Chyang W, Jyh-Ming L et al. Consensus on the clinical management, screening-to-treat, and surveillance of Helicobacter pylori infection to improve gastric cancer control on a nationwide scale. Helicobacter. 2017; 22:e12368.https:// doi.org/10.1111/hel.12368

21. Huang J-Q, Sridhar S, Chen Y, Hunt RH. Meta-analysis of the relationship between Helicobacter pylori seropositivity and gastric cancer. Gastroenterology 1998; 114: 1169-1179.

22. Helicobacter, Cancer Collaborative Group. Gastric cancer and Helicobacter pylori: a combined analysis of 12 case control studies nested within prospective cohorts. Gut 2001; 49: 347-353.

23. Uemura N, Okamoto $\mathrm{S}$, Yamamoto $\mathrm{S}$ et al. Helicobacter pylori infection and the development of gastric cancer. N Engl J Med 2001;345:784-789.

24. Hsu PI, Lai KH, Hsu PN et al. Helicobacter pylori infection and the risk of gastric malignancy. Am J Gastroenterol 2007; 102: 725-730. 
25. Huang JQ, Zheng GF, Sumanac K, Irvine EJ, Hunt RH. Metaanalysis of the relationship between cagA seropositivity and gastric cancer. Gastroenterology 2003; 125: 1636-1644.

26. Shibata A, Parsonnet J, Longacre TA et al. CagA status of Helicobacter pylori infection and $\mathrm{p} 53$ gene mutations in gastric adenocarcinoma. Carcinogenesis 2002; 23: 419-424.

27. Parkin DM. The global health burden of infection-associated cancers in the year 2002. Int J Cancer 2006; 118: 3030-3044.

28. F. Mégraud, E. Bessède and C. Varon.Helicobacter pylori infection and gastric carcinoma. Clin Microbiol Infect 2015;21: 984-990

29. Correa P, Haenszel W, Cuello C, Tannenbaum S, Archer M. A model for gastric cancer epidemiology. Lancet 1975;2(7924): $58-60$.

30. Hocker M, Rosenberg I, Xavier R et al. Oxidative stress activates the human histidine decarboxylase promoter in ags gastric cancer cells. J Biol Chem 1998; 273: 23046-23054.

31. Brenes F, Ruiz B, Correa P et al. Helicobacter pylori causes hyperproliferation of the gastric epithelium: pre- and posteradication indices of proliferating cell nuclear antigen. Am J Gastroenterol 1993; 88: 1870-1875.

32. Yamaoka Y, Kato M, Asaka M. Geographic differences in gastric cancer incidence can be explained by differences between Helicobacter pylori strains. Intern Med 2008; 47: 1077-1083.

33. Cuzick J, Otto F, Baron JA et al. Aspirin and non-steroidal antiinflammatory drugs for cancer prevention: an international consensus statement.Lancet Oncol 2009; 10: 501-507.

34. Correa P, Houghton J. Carcinogenesis of Helicobacter pylori. Gastroenterology 2007; 133: 659-672

35. Houghton J, Stoicov C, Nomura S et al. Gastric cancer originating from bone marrow-derived cells. Science 2004;306:1568-1571.

36. Giannakis M, Chen SL, Karam SM, Engstrand L, Gordon JI. Helicobacter pylori evolution during progression from chronic atrophic gastritis to gastric cancer and its impact on gastric stem cells. Proc Natl Acad Sci USA 2008;105: 4358-4363.

37. Lynch HT, Grady W, Suriano G, Huntsman D: Gastric cancer: new genetic developments. J Surg Oncol 2005; 90: 114-133; discussion 133 .

38. Ernst PB, Gold BD: The disease spectrum of Helicobacter pylori : the immunopathogenesis of gastroduodenal ulcer and gastric cancer. Annu Rev Microbiol 2000; 54: 615-640.

39. Kuper H, Adami HO, Trichopoulos D: Infections as a major preventable cause of human cancer. J Intern Med 2000; 248: 171-183.

40. Wei Zhang a Hong Lu a David Y. Graham. An Update on Helicobacter pylori as the Cause of Gastric Cancer. Gastrointest Tumors 2014;1:155-165. DOI: 10.1159/000365310

41. Ahmad MM, Rahman M, Rumi AK, Islam S, Huq F, Chowdhury MF, Jinnah F, Morshed MG, Hassan MS, Khan AK, Hasan
M.Prevalence of Helicobacter pylori in asymptomatic population — a pilot serological study in Bangladesh. J Epidemiol. 1997 Dec;7(4):251-4.

42. Mahalanabis D, Rahman MM, Sarker SA, et al. Helicobacter pylori infection in the young in Bangladesh: prevalence, socioeconomic and nutritional aspects. Int $\mathrm{J}$ Epidemiol 1996;25:894-8.

43. Kamangar F, Dawsey SM, Blaser MJ. Opposing risks of gastric cardia and noncardia gastric adenocarcinomas associated with Helicobacter pylori seropositivity. J Natl Cancer Inst 2006;98:1445-52.

44. Helicobacter and Cancer Collaborative Group. Gastric cancer and Helicobacter pylori: a combined analysis of 12 case control studies nested within prospective cohorts. Gut 2001;49:347-53.

45. Kato M, Asaka M, Shimizu Y, et al. Relationship between Helicobacter pylori infection and the prevalence, site and histological type of gastric cancer. Aliment Pharmacol Ther 2004;20:85-9.

46. Habib AM, Md, Alam MJ,Rudra B,Quader MA and Al-Forkan M. Analysis of Helicobacter pylori Prevalence in Chittagong, Bangladesh, Based on PCR and CLO Test. Microbiology Insights 2016:947-50 doi:10.4137/MBI.S39858.

47. Sarker KW , Md.Kabir MJ,A.K.M.BhuyianAKM M,Md. Shahjadul Alam Chowdhury FR,Ahad MA, Rahman MA, Rahman MM. H. pylori infection and gastric cancer in Bangladesh: a case-control study.International Journal of Surgery Oncology (2017) 2:e44

48. Lee Y-C, Chiang T, Liou JM, et al. Mass eradication of Helicobacter pylori to prevent gastric cancer: theoretical and practical considerations. Gut and Liver 2016;10:12-26.

49. Akiko Shiotania, Putao Cenb, David Y. Grahamc Eradication of gastric cancer is now both possible and practical.Seminars in Cancer Biology 2013; 23:492-501.

50. Maekita T, Nakazawa K, Mihara M, Nakajima T, Yanaoka K, Iguchi $\mathrm{M}$, et al.High levels of aberrant DNA methylation in Helicobacter pylori-infected gastricmucosae and its possible association with gastric cancer risk. Clinical Cancer Research 2006;12:989-95.

51. Yi-Chia L, Hsiu-Hsi TC, Han-Mo C, Chia-Tung S,Hung C, TzengYing L,Ming-Shiang W, Jaw-Town L.The benefit of mass eradication of Helicobacter pylori infection: a community-based study of gastric cancer prevention.Gut 2013;62: 676-82. doi:10.1136/gutjnl-2012-302240

52. Yi-Chia L, Tsung-Hsien C, Jyh-Ming L, Hsiu-Hsi C, Ming-Shiang W, and David Y G. Mass Eradication of Helicobacter pylori to Prevent Gastric Cancer: Theoretical and Practical Considerations. Gut and Liver 2016;10: 12-26. 